\title{
Phosphine Sulfide-Based Bipolar Host Materials for Blue Phosphorescent Organic Light-Emitting Diodes
}

\author{
Gaozhan Xie, Jiangchao Wang, Yang Cao, Xudong Xue, Xiao Zhang, Chang Liu, Huanhuan Li *, Ye Tao * \\ and Runfeng Chen
}

check for updates

Citation: Xie, G.; Wang, J.; Cao, Y.; Xue, X.; Zhang, X.; Liu, C.; Li, H.; Tao, Y.; Chen, R. Phosphine Sulfide-Based Bipolar Host Materials for Blue Phosphorescent Organic Light-Emitting Diodes. Molecules 2021, 26, 4079. https://doi.org/ $10.3390 /$ molecules 26134079

Academic Editor: Roy

Man-Keung Fung

Received: 29 May 2021

Accepted: 1 July 2021

Published: 3 July 2021

Publisher's Note: MDPI stays neutral with regard to jurisdictional claims in published maps and institutional affiliations.

Copyright: (c) 2021 by the authors. Licensee MDPI, Basel, Switzerland. This article is an open access article distributed under the terms and conditions of the Creative Commons Attribution (CC BY) license (https:/ / creativecommons.org/licenses/by/ $4.0 /)$.
State Key Laboratory of Organic Electronics and Information Displays \& Institute of Advanced Materials (IAM), Jiangsu National Synergistic Innovation Center for Advanced Materials (SICAM), Nanjing University of Posts \& Telecommunications, 9 Wenyuan Road, Nanjing 210023, China; iamgzxie@njupt.edu.cn (G.X.); 1220066216@njupt.edu.cn (J.W.); 1019061401@njupt.edu.cn (Y.C.); 1020062012@njupt.edu.cn (X.X.); 18805197992@163.com (X.Z.); 1020061916@njupt.edu.cn (C.L.); iamrfchen@njupt.edu.cn (R.C.)

* Correspondence: iamhhli@njupt.edu.cn (H.L.); iamytao@njupt.edu.cn (Y.T.)

Abstract: Three phosphine sulfide-based bipolar host materials, viz CzPhPS, DCzPhPS, and TCzPhPS were facilely prepared through a one-pot synthesis in excellent yields. The developed hosts exhibit superior thermal stabilities with the decomposition temperatures $\left(T_{\mathrm{d}}\right)$ all exceeding $350{ }^{\circ} \mathrm{C}$ and the melting temperatures $\left(T_{\mathrm{m}}\right)$ over $200{ }^{\circ} \mathrm{C}$. In addition, their triplet energy $\left(E_{\mathrm{T}}\right)$ levels are estimated to be higher than $3.0 \mathrm{eV}$, illustrating that they are applicable to serve as hosts for blue phosphorescent organic light-emitting diodes (PhOLEDs). The maxima luminance, current efficiency (CE), power efficiency (PE), and external quantum efficiency (EQE) of $17,223 \mathrm{~cd} \mathrm{~m}^{-2}, 36.7 \mathrm{~cd} \mathrm{~A}^{-1}, 37.5 \mathrm{~lm} \mathrm{~W}^{-1}$, and $17.5 \%$ are achieved for the blue PhOLEDs hosted by CzPhPS. The PhOLEDs based on DCzPhPS and TCzPhPS show inferior device performance than that of CzPhPS, which might be ascribed to the deteriorated charge transporting balance as the increased number of the constructed carbazole units in DCzPhPS and TCzPhPS molecules would enhance the hole-transporting ability of the devices to a large extent. Our study demonstrates that the bipolar hosts derived from phosphine sulfide have enormous potential applications in blue PhOLEDs, and the quantity of donors should be well controlled to exploit highly efficient phosphine sulfide-based hosts.

Keywords: bipolar host material; phosphorescent organic light-emitting diode; phosphine sulfide; carbazole; triplet energy level

\section{Introduction}

Phosphorescent organic light-emitting diodes (PhOLEDs) have been extensively researched in recent decades since they can achieve an internal quantum efficiency of nearly $100 \%$ by harvesting both singlet and triplet excitons, and have the advantages of excellent device stability, high color purity, as well as flexible color adjustment [1-3]. Nevertheless, the emitters in PhOLEDs need to be incorporated into appropriate hosts for the reason that the high concentration of emitters can result in concentration quenching, such as triplet-triplet annihilation (TTA) and triplet-singlet annihilation (TSA) [4,5]. As a consequence, it is of extreme importance to exploit the state-of-the-art hosts to attain high -performance PhOLEDs.

Bipolar hosts, generally prepared by combining the donor (D) and acceptor (A) units into a molecular skeleton (i.e., D-A molecules), are capable of achieving a broad recombination zone and balanced charge in the emitting layer (EML), so they are considered one of the most promising types of hosts to realize highly efficient PhOLEDs [6-8]. Up until the present, the bipolar hosts for green, yellow, and red phosphorescent emitters have made commendable progress [9-12], while it is still challenging to develop superior blue hosts because the blue phosphors inherently possess high triplet energy $\left(E_{\mathrm{T}}\right)$ levels [13-15], thus the $E_{\mathrm{T}}$ of the hosts should be accordingly elevated to prevent the reverse energy 
transfer from the guests back to the hosts and to effectively confine triplet excitons on the guests. However, the non-negligible intramolecular interactions in D-A molecules would produce a low energy charge transfer $(\mathrm{CT})$ state, which brings down the $E_{\mathrm{T}}$ levels [16]. Therefore, for the design of blue bipolar hosts that exhibit the balanced charge transport ability simultaneously maintaining a high $E_{\mathrm{T}}, \mathrm{D}$ and $\mathrm{A}$ constructed groups should be meticulously selected.

Aryl phosphine oxide (APO, Scheme 1), with moderated electron-withdrawing ability and excellent charge transport properties, has been widely applied in blue bipolar hosts [17-24]. Through simply replacing the $\mathrm{O}$ atom of APO with the $\mathrm{S}$ atom, aryl phosphine sulfide (APS, Scheme 1) can be gained. Since the ${ }^{3} p$ lone-pair electrons of $S$ exhibit the larger scope than the ${ }^{2} p$ lone-pair electrons of $\mathrm{O}$, the sulfur atom and $\pi$-system interactions (S- $\pi$ interactions) can be formed, which would partly enhance intramolecular electronic communication, leading to selectively modulated frontier molecular orbital energy levels and improved electrical performance $[25,26]$. On the other hand, APO with stronger polarization of $\mathrm{P}=\mathrm{O}$ exhibits weaker intramolecular electronic coupling and an unduly deep highest occupied molecular orbital (HOMO) energy level, rendering it difficult to maintain the efficient and balanced charge injection and transportation. Hence, the hosts consisting of APS tend to have enhanced electrical performance than that of APO. However, the APS-based hosts have hardly been reported so far, and need to be further exploited. In 2016, our group reported a variety of remarkably high-performance bipolar hosts adopting the phenylphosphine sulfide as the electron-withdrawing unit, of which the host named as DNCzPS ((9H-carbazol-9-yl)diphenylphosphine sulfide, Scheme 1$)$ displays a $E_{\mathrm{T}}$ level as high as $2.97 \mathrm{eV}$ [25]. The blue PhOLED based on DNCzPS turns on at $2.9 \mathrm{eV}$ with an impressive maximum external quantum efficiency (EQE) of $21.7 \%$. Subsequently, we prepared the APS-based host DPSSi ((9-(4-(triphenylsilyl)phenyl)-9H-carbazole-3,6-diyl) bis(diphenyl-phosphine sulfide), Scheme 1), whose blue PhOLED shows a maximum EQE of $20.8 \%$, which is 1.58 folds of enhancement compared to that of the APO-based host DPOSi ((9-(4-(triphenylsilyl)phenyl)-9H-carbazole-3,6-diyl)bis(diphenylphosphine oxide), Scheme 1) in the same device structure [26]. Encouraged by the above-mentioned results, in this contribution we employ triphenylphosphine sulfide as A group, and select mono-, di-, as well as tri-substituted carbazoles with tunable hole-transport ability as D units, respectively, to construct three original bipolar hosts, i.e., CzPhPS, DCzPhPS, and TCzPhPS. All three compounds possess the high $E_{\mathrm{T}} \mathrm{S}$ of $\sim 3.0 \mathrm{eV}$, much higher than those of the commonly used blue emitters, demonstrating that they are promising candidates as the hosts for blue OLEDs. The blue PhOLED hosted by CzPhPS achieves a low turn-on voltage of $3.1 \mathrm{eV}$ and a maximum EQE of $17.5 \%$, better than the device performance of DCzPhPS and TCzPhPS, which could be owed to its better charge transport balance. 


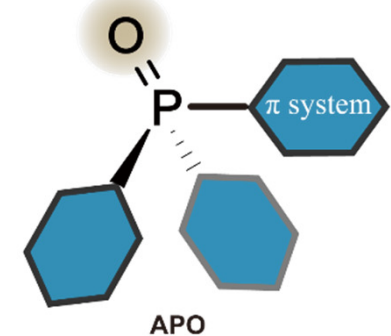

APO

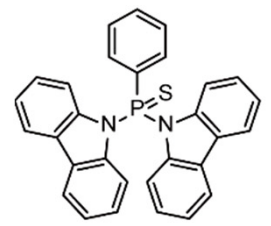

DNCzPS

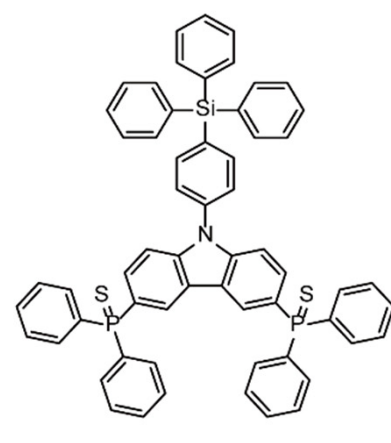

DPSSi

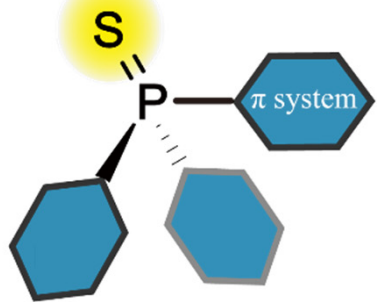

APS

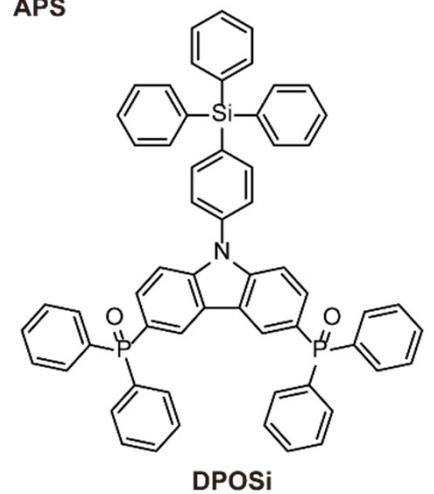

Scheme 1. Chemical structures of APO, APS, DNCzPS, DPSSi, and DPOSi.

\section{Results and Discussion}

\subsection{Material Synthese}

CzPhPS, DCzPhPS, and TCzPhPS were facilely obtained by a one-pot synthesis as outlined in Scheme 2. A nucleophilic addition reaction of the organolithium compounds formed from 9-(4-bromophenyl)-9H-carbazole and $n$-BuLi followed by the treatment with sublimed sulfur in dichloromethane furnished the three target molecules in 57-63\% yields. Their molecular structures were firmly confirmed by ${ }^{1} \mathrm{H}$ and ${ }^{13} \mathrm{C}$ nuclear magnetic resonance (NMR) spectra, as well as high-resolution mass spectra (HRMS) (Supporting Information, Figures S1-S9).

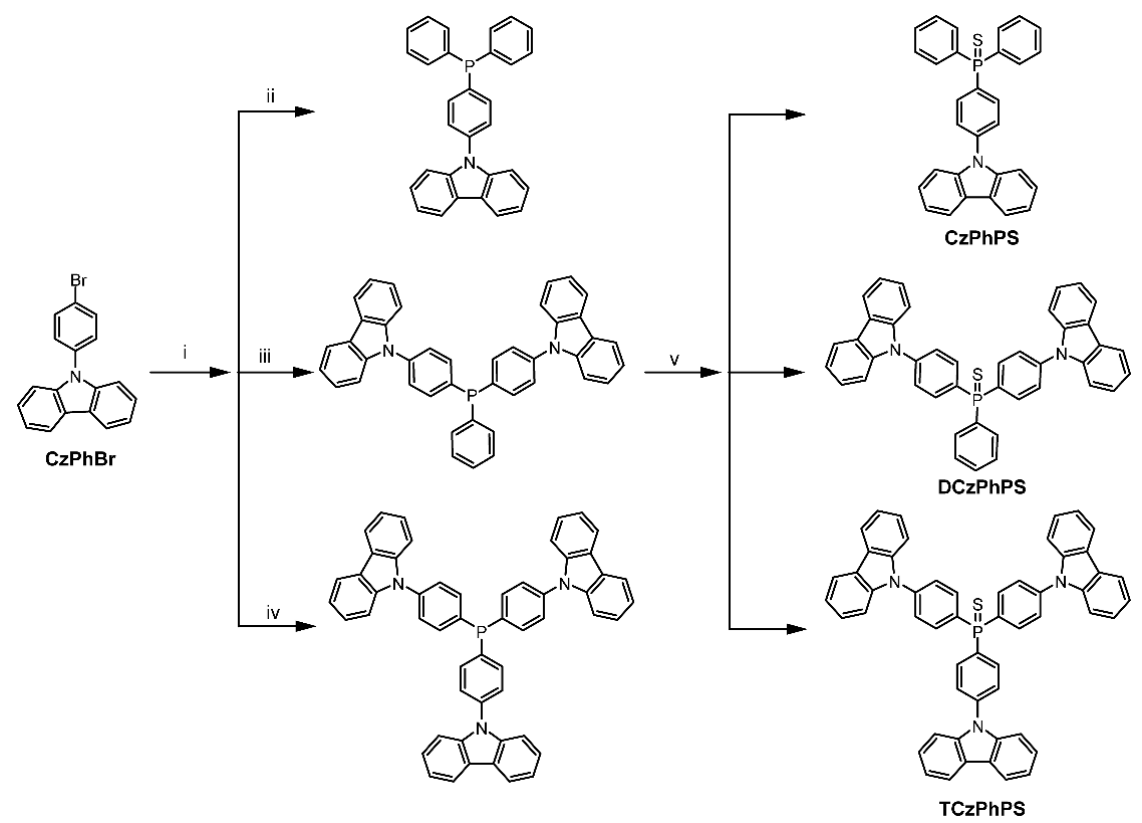

Scheme 2. Synthetic routes to CzPhPS, DCzPhPS, and TCzPhPS. (i) $n$-BuLi, THF, $-78{ }^{\circ} \mathrm{C}, 2 \mathrm{~h}$; (ii) $\mathrm{Ph}_{2} \mathrm{PCl},-78{ }^{\circ} \mathrm{C}, 1 \mathrm{~h}$; (iii) $\mathrm{PhPCl}_{2},-78{ }^{\circ} \mathrm{C}, 1 \mathrm{~h}$; (iv) $\mathrm{P}\left(\mathrm{OCH}_{2} \mathrm{CH}_{3}\right)_{3},-78{ }^{\circ} \mathrm{C}, 1 \mathrm{~h}$; (v) Sulfur, $\mathrm{CH}_{2} \mathrm{Cl}_{2}$, room temperature (r.t.), overnight. 


\subsection{Thermal Stability}

In order to investigate the thermal properties of the designed phosphine sulfide molecules, the thermal gravimetric analysis (TGA) and differential scanning calorimetry (DSC) measurements were carried out under a nitrogen atmosphere. As shown in Figure 1a and Table 1, all three compounds exhibit outstanding thermal stability with the decomposition temperatures $\left(T_{\mathrm{d}}\right)$ of 366,447 , and $479^{\circ} \mathrm{C}$ for CzPhPS, DCzPhPS, and TCzPhPS, respectively, indicating that $\mathrm{P}=\mathrm{S}$ bonds are stable enough for the evaporation process to fabricate OLEDs. In addition, for these compounds, no glass transition temperature $\left(T_{\mathrm{g}}\right)$ is detected in the tested temperature range shown in Figure $1 \mathrm{~b}$, while their melting temperatures $\left(T_{\mathrm{m}}\right)$ can be clearly observed with the $T_{\mathrm{m}}$ values all exceeding $200^{\circ} \mathrm{C}$. From CzPhPS to TCzPhPS, as expected, the phase stability becomes better due to the increased number of carbazole units.

(a)

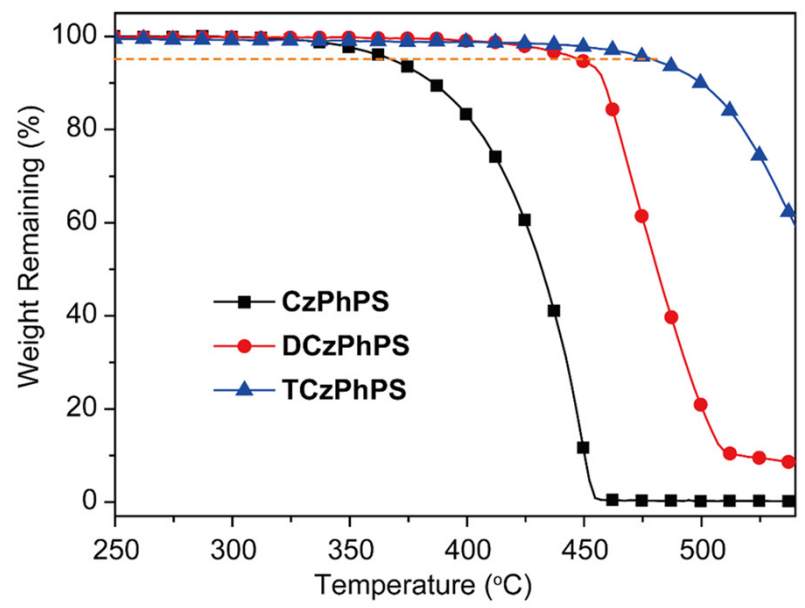

(b)
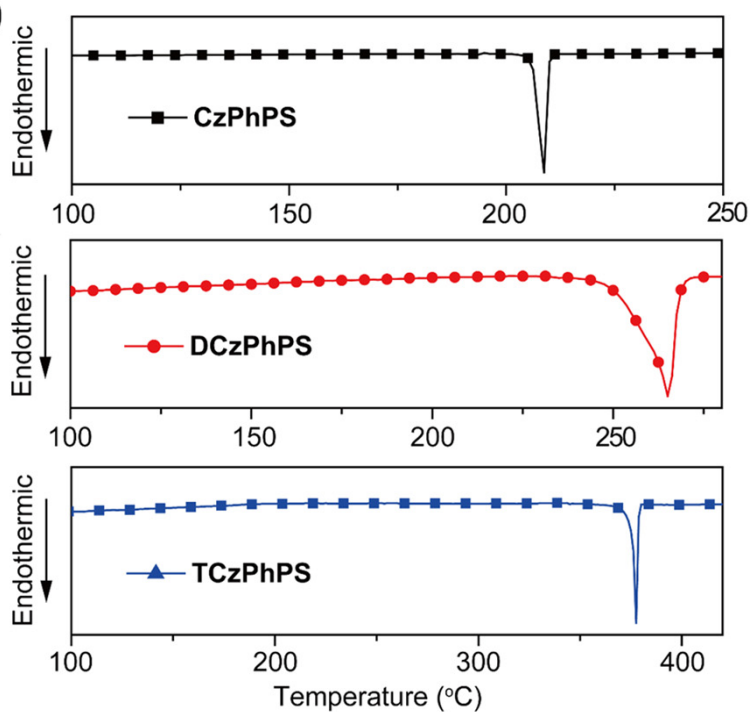

Figure 1. TGA (a) and DSC (b) curves of CzPhPS, DCzPhPS, and TCzPhPS.

Table 1. Thermal, photophysical, electrochemical properties, and energy levels of the developed compounds.

\begin{tabular}{|c|c|c|c|c|c|c|c|c|c|}
\hline Comp. & $\underset{\left({ }^{\circ} \mathrm{C}\right)}{T_{\mathrm{d}}[\mathrm{a}] / T_{\mathrm{m}}[\mathrm{b}]}$ & $\underset{(\mathrm{nm})}{\lambda_{\mathrm{abs}}[\mathrm{c}]}$ & $\begin{array}{c}\lambda_{\text {em }}[\mathrm{c}] \\
(\mathrm{nm})\end{array}$ & $\underset{(\%)}{\text { PLQY [d] }}$ & $\underset{\text { (ns) }}{\text { Lifetime }[d]}$ & $\begin{array}{c}\text { HOMO (eV) } \\
\text { Meas. [e]/ } \\
\text { Calcd [f] }\end{array}$ & $\begin{array}{l}\text { LUMO (eV) } \\
\text { Meas. [g]/ } \\
\text { Calcd [f] }\end{array}$ & $\begin{array}{c}E_{\mathrm{g}}(\mathrm{eV}) \\
\text { Meas. [h]/ } \\
\text { Calcd [f] }\end{array}$ & $\begin{array}{c}E_{\mathrm{T}}(\mathrm{eV}) \\
\text { Meas. [i]/ } \\
\text { Calcd [f] }\end{array}$ \\
\hline CzPhPS & $366 / 209$ & $298,332,343$ & 378 & 24 & 2.11 & $-5.71 /-5.49$ & $-2.29 /-1.12$ & $3.43 / 4.37$ & $3.00 / 2.98$ \\
\hline DCzPhPS & $447 / 264$ & $298,335,343$ & 381 & 20 & 1.99 & $-5.70 /-5.52$ & $-2.28 /-1.23$ & $3.42 / 4.29$ & $3.01 / 2.98$ \\
\hline TCzPhPS & $479 / 377$ & $298,335,344$ & 380 & 22 & 2.06 & $-5.74 /-5.56$ & $-2.34 /-1.29$ & $3.38 / 4.27$ & $3.01 / 2.99$ \\
\hline
\end{tabular}

[a] Decomposition temperatures at $5 \mathrm{wt} \%$ weight loss; [b] Melting temperatures; [c] Absorption and PL peaks measured in solid thin films; [d] Measured in $10^{-5} \mathrm{M}$ dichloromethane solution; [e] HOMO energy levels estimated from the onset potentials of the oxidation peaks in cyclic voltammograms; [f] Calculated from Gaussian $09 \mathrm{M} 062 X ; \mathrm{g}]$ Obtained from $E_{\mathrm{HOMO}}$ and $E_{\mathrm{g}}$ according to the equation of $E_{\mathrm{LUMO}}=E_{\mathrm{g}}+E_{\mathrm{HOMO}} ;[\mathrm{h}]$ Estimated from the absorption edge of thin films $\left(\lambda_{\text {onset }}\right)$ employing the formula of $E_{\mathrm{g}}=1240 / \lambda_{\text {onset }} ;[\mathrm{i}]$ Estimated from the first peaks of phosphorescence spectra recorded in tetrahydrofuran at $77 \mathrm{~K}$.

\subsection{Photophysical Properties}

Figure 2a displays the absorption and photoluminescence (PL) spectra of the developed materials in solid thin films. CzPhPS, DCzPhPS, and TCzPhPS exhibit quite similar absorption and emission spectra as they are constructed by the same electron-accepting unit (triphenyl phosphine sulfide) and electron-donating unit (carbazole), and the quantities of carbazole units do not have a large effect on their intermolecular charge transfer properties. All three compounds exhibit a sharp absorption peak at $298 \mathrm{~nm}$, which can be ascribed to the $\pi-\pi^{*}$ transitions of carbazole groups [27], whereas the absorption peaks of around 335 and $343 \mathrm{~nm}$ might be due to the intramolecular CT from the electron donor carbazole to the electron acceptor triphenyl phosphine sulfide. CzPhPS, DCzPhPS, and TCzPhPS display 
similar PL spectra with the emission peaks at 378,381 , and $380 \mathrm{~nm}$, respectively. In addition, all the time-resolved transient PL decay curves of those pure films shown in Figure $2 b$ consist of a nanosecond-order lifetime of $\sim 2 \mathrm{~ns}$, suggesting their fluorescent nature. To further investigate the luminescent properties of the target molecules, their photoluminescence quantum yields (PLQYs) were also measured in $10^{-5} \mathrm{M}$ dichloromethane solution. CzPhPS, DCzPhPS, and TCzPhPS exhibit low PLQYs of $24 \%, 20 \%$, and $22 \%$, respectively. Moreover, according to the first peaks of phosphorescence spectra (see Figure S10) recorded at $77 \mathrm{~K}$ in THF with a delay time of $20 \mathrm{~ms}$, the $E_{\mathrm{T}} \mathrm{s}$ of CzPhPS, DCzPhPS, and TCzPhPS are estimated to be $\sim 3.0 \mathrm{eV}$, which is high enough for them to serve as hosts for commonly used blue emitters such as bis(3,5-difluoro-2-(2-pyridyl)phenyl-(2-carboxypyridyl)iridium (III) (FIrpic, $E_{\mathrm{T}}: 2.65 \mathrm{eV}$ ) [28].

(a)

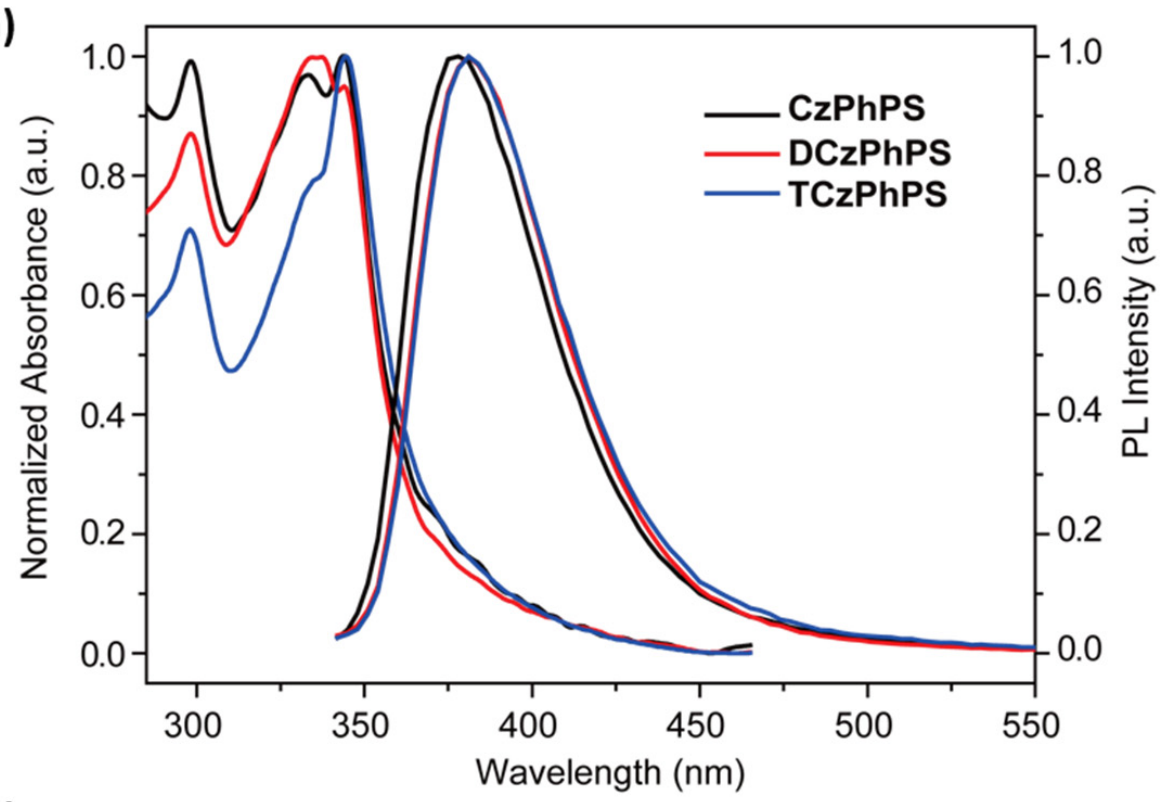

(b)

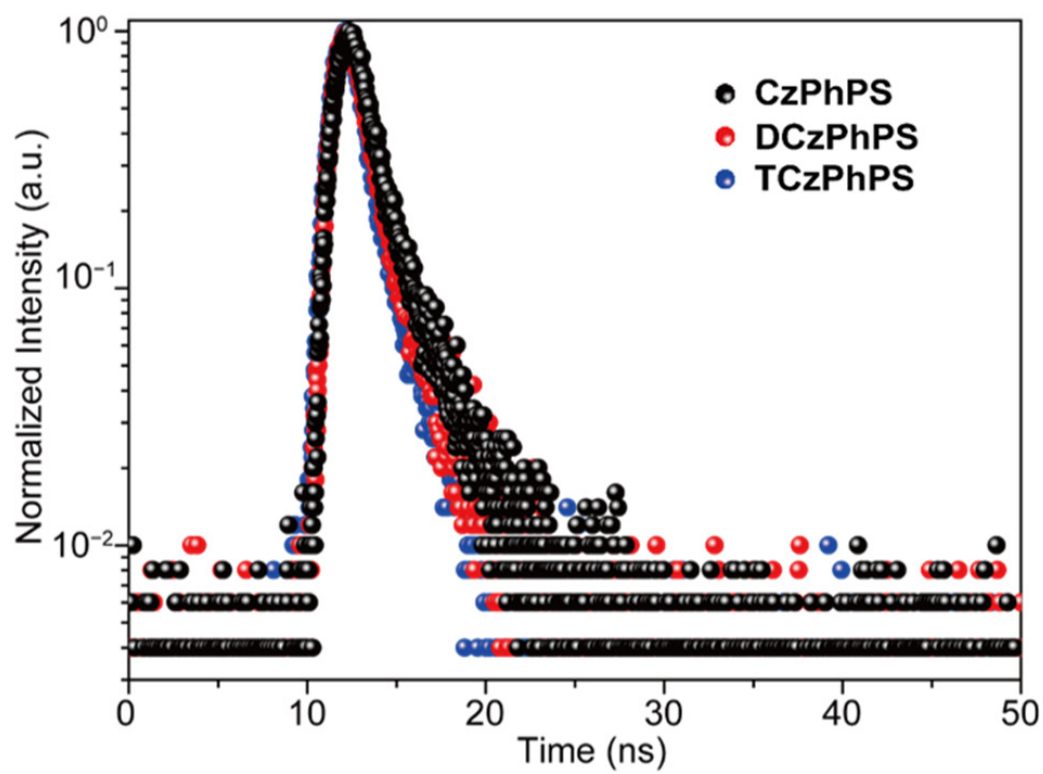

Figure 2. Absorption and PL spectra (a), and time-resolved transient PL decay curves (b) of CzPhPS, DCzPhPS, and TCzPhPS in solid thin films. 


\subsection{Electrochemical Properties}

Cyclic voltammetry (CV) measurements were carried out to obtain the electrical properties of these phosphine sulfide compounds [29]. As shown in Figure 3, all the compounds exhibit an irreversible oxidative wave with the onset potential at $\sim 0.95 \mathrm{eV}$, attributed to the oxidation of carbazole units, whereas their reduced waves can not be observed when the negative voltage is applied. The HOMO energy levels are estimated to be $\sim-5.72 \mathrm{eV}$ based on the onset potential of the oxidative wave, and their lowest unoccupied molecular orbital (LUMO) energy levels are calculated to be $-2.29,-2.28$, and $-2.34 \mathrm{eV}$ for CzPhPS, DCzPhPS, and TCzPhPS, respectively, according to the HOMO energy levels and the absorption edge of thin films.

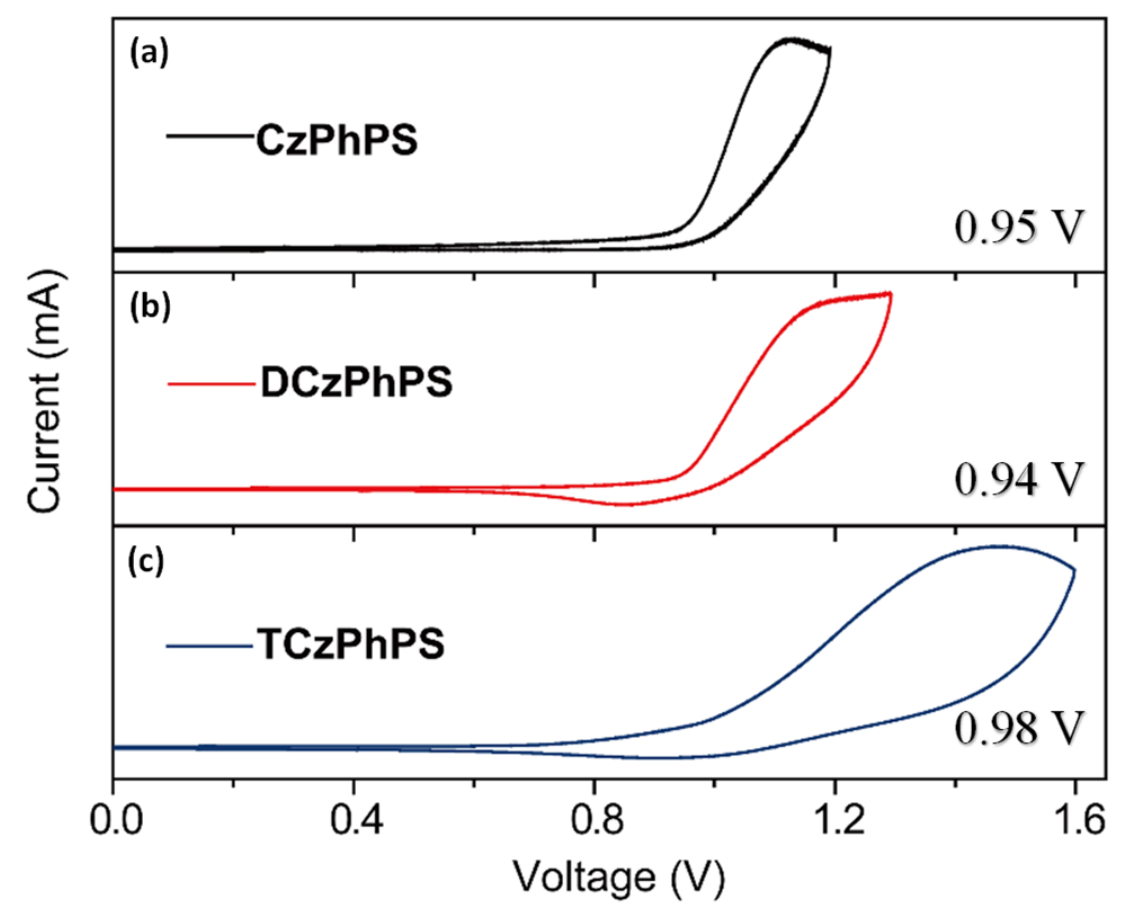

Figure 3. Cyclic voltammograms of (a) CzPhPS, (b) DCzPhPS, and (c) TCzPhPS films deposited on the working electrodes.

\subsection{Theoretical Investigations}

Density-functional theory (DFT) calculations were further performed to acquire insight into the structure-property relationships of CzPhPS, DCzPhPS, and TCzPhPS [30]. As shown in Figure 4, the LUMO of these three compounds is predominantly located on the triphenylphosphine sulfide group due to its electron-withdrawing feature, while the HOMO is mainly distributed on electron-donating carbazole units, as expected. The separated HOMO and LUMO distributions indicate that the developed compounds should be of bipolar charge carrier transporting ability, which are suitable for being served as bipolar hosts in OLEDs. In addition, the HOMO/LUMO energy levels are simulated to be $-5.49 /-1.12 \mathrm{eV},-5.52 /-1.23 \mathrm{eV}$, and $-5.56 /-1.29 \mathrm{eV}$ for CzPhPS, DCzPhPS, and TCzPhPS, respectively, calculated at the TD-DFT/M062X level using Gaussian 09W software. The trend is fairly in line with the above-mentioned CV measurements. In addition, the computational $E_{\mathrm{T}}$ levels are almost the same with experimental results evaluated from phosphorescent spectra with the values of $\sim 3.0 \mathrm{eV}$. 


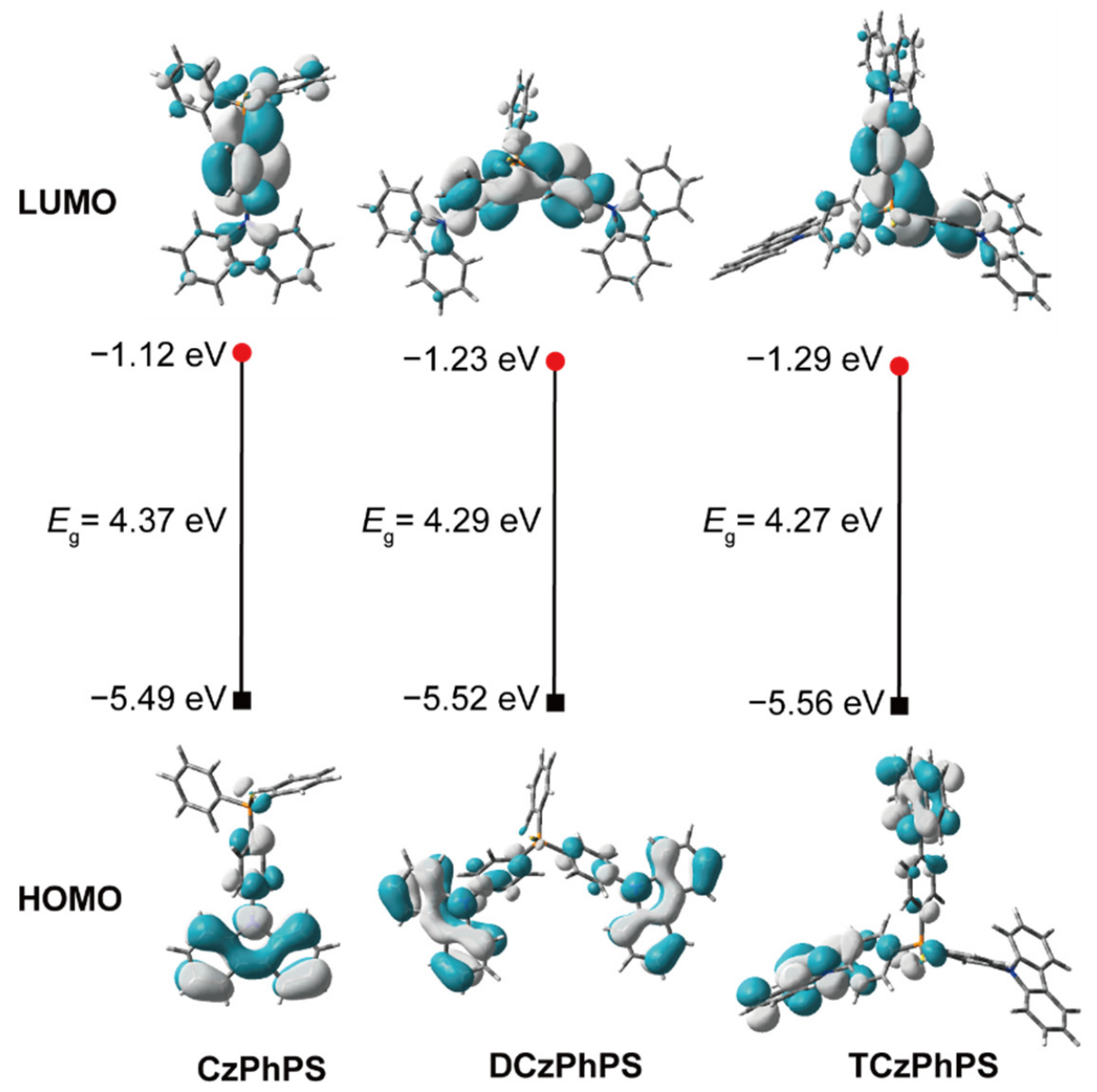

Figure 4. HOMO and LUMO distributions of the developed molecules calculated at the TDDFT/M062X level by Gaussian 09W.

\subsection{Device Fabrication and Performance}

In light of the high $E_{\mathrm{T}}$ energy levels and excellent bipolar charge transporting ability of the developed phosphine sulfide materials, the blue PhOLEDs hosted by CzPhPS (Device A), DCzPhPS (Device B), and TCzPhPS (Device C) were fabricated in a typical architecture of indium tin oxide (ITO)/poly(3,4-ethylenedioxythiophene):poly(styrenesulfonate) (PEDOT:PSS) (30 nm)/1,3,5-triazo-2,4,6-triphosphorine-2,2,4,4,6,6-tetrachloride (TAPC) $(20 \mathrm{~nm}) /$ host: $25 \mathrm{wt} \%$ FIrpic (20 nm)/1,3,5-tri $(m$-pyrid-3-yl-phenyl) benzene (TmPyPB) $(35 \mathrm{~nm})$ /lithium fluoride $(\mathrm{LiF})(1 \mathrm{~nm}) / \mathrm{Al}(100 \mathrm{~nm})$. The energy level diagrams and chemical structures of the adopted materials in the blue PhOLEDs are displayed in Figure 5a,b. Given that the HOMO levels of all three host materials are just $0.2 \mathrm{eV}$ deeper-lying than that of TAPC, there should be no hole-injection barrier between HTL and EML [31]. Likewise, there should be no problem for electrons to jump from ETL to EML as their LUMO energy gap is only $0.4 \mathrm{eV}$. Luminance-voltage-current density characteristics, EL spectra, and efficiencies-luminance curves of the devices are shown in Figure $5 c, d$; the corresponding data are listed in Table 2. Device A turns on at a low voltage of $3.1 \mathrm{eV}$ with a peak wavelength, maxima luminance, current efficiency (CE), power efficiency (PE), and EQE of $472 \mathrm{~nm}, 17,223 \mathrm{~cd} \mathrm{~m}^{-2}, 36.7 \mathrm{~cd} \mathrm{~A}^{-1}, 37.5 \mathrm{~lm} \mathrm{~W}^{-1}$, and 17.5\%. Notably, Device B and Device $\mathrm{C}$ exhibit inferior device performance with maxima EQE of $16.1 \%$ and $7.2 \%$, respectively. It is well known that carrier transporting balance is of particular importance to render the holes, and electrons recombine in the emitting layer so that high-performance OLEDs can be achieved [32]. In addition, introducing more carbazole groups into molecule skeletons would increase the hole-transporting ability of the compounds [33-35]. Hence, it is rational to deduce that the poorer device performances of Device B and Device $\mathrm{C}$ might be 
ascribed to the increased number of carbazoles in the host molecules, which enhance the hole-transporting ability of the devices, thus deteriorating the carrier transporting balance.

(a)

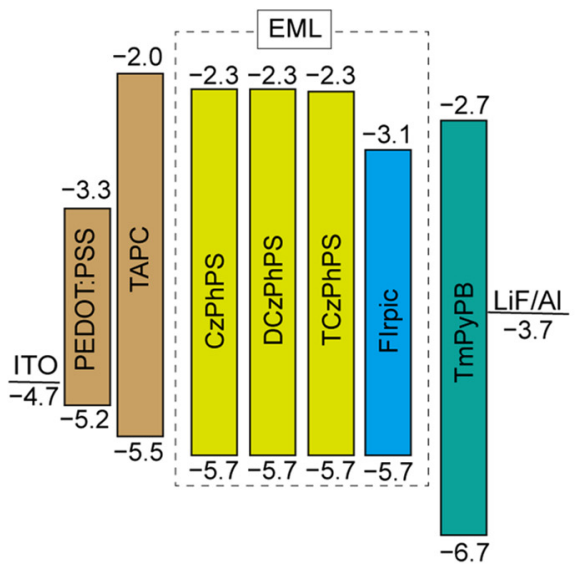

(c)

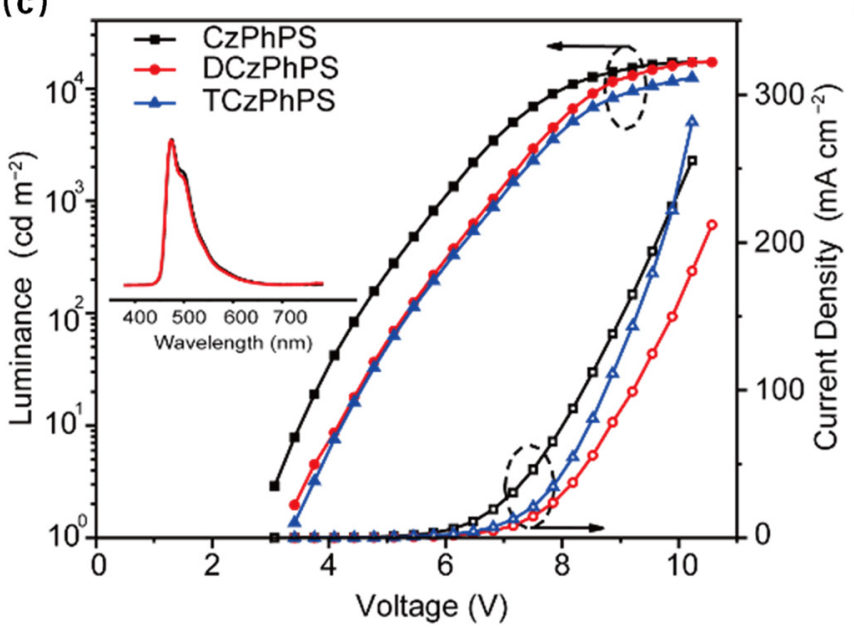

(b)<smiles>Cc1ccc(N(c2ccc(C)cc2)c2ccc(C3(c4ccc(C)cc4)CCCCC3)cc2)cc1</smiles>

TAPC<smiles>O=C1OC(n2cccc2)(n2cccc2-c2ccccc2-c2ccc(F)cc2F)n2cccc21</smiles>

FIrpic

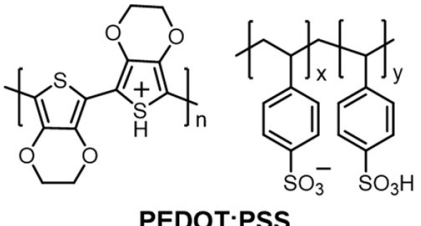

PEDOT:PSS

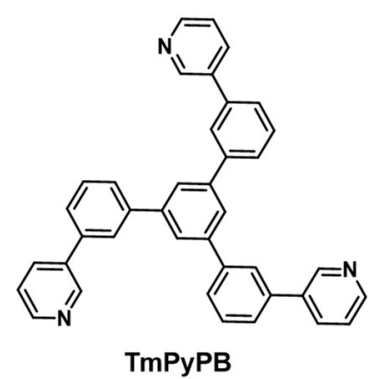

(d)

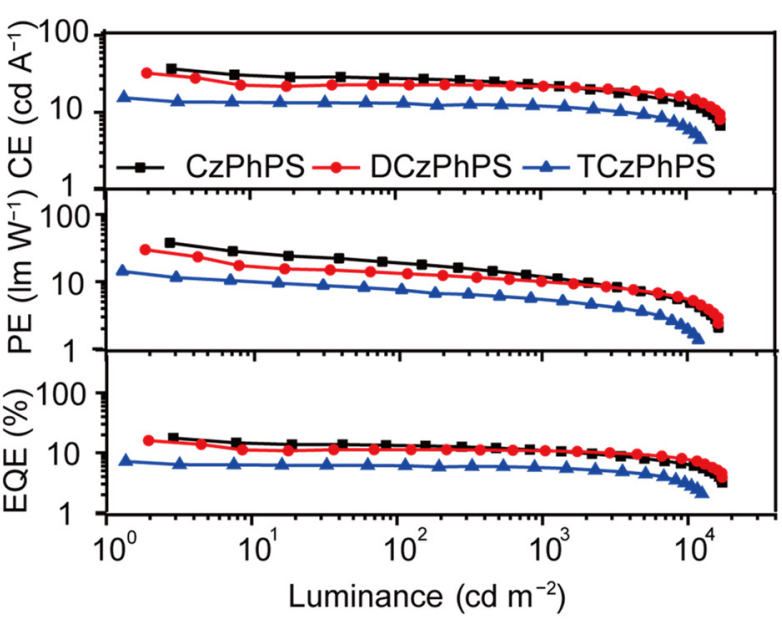

Figure 5. Energy level diagrams (a) and chemical structures (b) of the materials used in the devices. Luminance-voltagecurrent density (c) and efficiencies-luminance (d) curves of the blue PhOLEDs devices. Inset: EL spectra at $5 \mathrm{~V}$.

Table 2. Electroluminescence (EL) performance of devices based on CzPhPS, DCzPhPS, and TCzPhPS hosts.

\begin{tabular}{|c|c|c|c|c|c|c|c|}
\hline Device & Host & $\begin{array}{c}\mathrm{V}_{\text {on }[\mathrm{a}]} \\
\left({ }^{\circ} \mathrm{C}\right)\end{array}$ & $\begin{array}{l}\lambda[b] \\
(\mathrm{nm})\end{array}$ & $\begin{array}{l}\text { Luminance [c] } \\
\quad\left(\mathrm{cd} \mathrm{m}^{-2}\right)\end{array}$ & $\begin{array}{c}\mathrm{CE}[\mathrm{d}] \\
\left(\operatorname{cd~} \mathrm{A}^{-1}\right)\end{array}$ & $\begin{array}{c}\mathrm{PE}[\mathrm{e}] \\
\left(\operatorname{lm} \mathrm{W}^{-1}\right)\end{array}$ & $\begin{array}{c}\text { EQE [f] } \\
(\%)\end{array}$ \\
\hline A & CzPhPS & 3.1 & 472 & 17,223 & 36.7 & 37.5 & 17.5 \\
\hline B & DCzPhPS & 3.4 & 472 & 17,176 & 32.3 & 29.8 & 16.1 \\
\hline $\mathrm{C}$ & TCzPhPS & 3.4 & 476 & 12,500 & 15.4 & 14.2 & 7.2 \\
\hline
\end{tabular}

[a] Turn-on voltage at a brightness of $1 \mathrm{~cd} \mathrm{~m}^{-2}$; [b] Electroluminescence peak wavelength at a voltage of $5 \mathrm{~V}$; [c] Maximum luminance; [d] Maximum current efficiency; [e] Maximum power efficiency; [f] Maximum external quantum efficiency.

\section{Materials and Methods}

\subsection{General Methods}

${ }^{1} \mathrm{H}$ and ${ }^{13} \mathrm{C}$-NMR spectra were recorded on a Bruker Ultra Shield Plus $400 \mathrm{MHz}$ instrument using $\mathrm{CDCl}_{3}$ as the solvent and tetramethylsilane (TMS) as the internal standard. The quoted chemical shifts are in $p p m$ and the $J$ values are expressed in $\mathrm{Hz}$. The splitting patterns have been designed as follows: $s$ (singlet), $d$ (doublet), $t$ (triplet), dd (doublet of doublets), and $\mathrm{m}$ (multiplet). HRMS were recorded on a LCT Premier XE (Waters) mass 
spectrometer. TGA measurements were conducted on a Netzsch STA2500 thermogravimet-

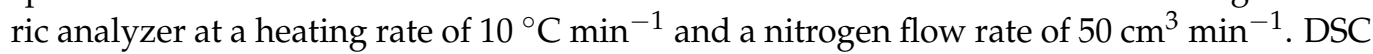
analyses were performed on a Netzsch DSC214 Polyma instrument under a heating rate of

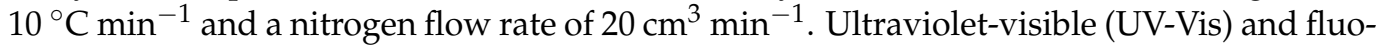
rescence spectra were recorded on a Jasco V-750 spectrophotometer and Edinburgh FLS980, respectively. The thin films for UV-Vis spectroscopy measurements were prepared by high-vacuum thermal evaporation at a rate of $0.1-0.2 \mathrm{~nm} \mathrm{~s}^{-1}$. The phosphorescence spectra of the compounds in $\mathrm{CH}_{2} \mathrm{Cl}_{2}$ were measured using a time-resolved Edinburgh FLS980 fluorescence spectrophotometer at $77 \mathrm{~K}$, with a $20 \mathrm{~ms}$ delay time using a microsecond flash lamp. CV measurements were performed at room temperature on a CHI660E system in a typical three-electrode cell with a working electrode (glass carbon), a reference electrode $\left(\mathrm{Ag} / \mathrm{Ag}^{+}\right.$, referenced against ferrocene/ferrocenium (FOC)), and a counter electrode ( $\mathrm{Pt}$ wire) in an acetonitrile solution of tetrabutylammonium hexafluorophosphate $\left(\mathrm{Bu}_{4} \mathrm{NPF}_{6}\right)$ $(0.1 \mathrm{M})$ at a sweeping rate of $100 \mathrm{mV} \mathrm{s}^{-1}$. The thin solid films of the developed molecules for $\mathrm{CV}$ measurements were formed by adding their solution dropwise on the surface of the glassy carbon working electrode followed by drying under ambient conditions. The $\mathrm{HOMO}$ energy levels $\left(E_{\mathrm{HOMO}}\right)$ of the materials were measured according to the reference energy level of ferrocene ( $4.8 \mathrm{eV}$ below the vacuum) as illustrated in Equation (1):

$$
E_{\mathrm{HOMO}}=-\left[E_{\text {onset }}^{\mathrm{ox}}-E_{\left(\mathrm{Fc} / \mathrm{Fc}^{+}\right)}+4.8\right] \mathrm{eV}
$$

where $E_{\left(\mathrm{Fc} / \mathrm{Fc}^{+}\right)}$is the onset oxidative voltage of FOC vs. $\mathrm{Ag} / \mathrm{Ag}^{+}$whose value is $0.4 \mathrm{~V}$ in our testing conditions, and $E_{\text {onset }}^{\text {ox }}$ is the onset potential of the oxidation wave. The LUMO energy level ( $\left.E_{\mathrm{LUMO}}\right)$ was estimated by adding the optical band-gap $\left(E_{\mathrm{g}}\right)$ to the corresponding HOMO energy level as in Equation (2):

$$
E_{\mathrm{LUMO}}=-\left[E_{\mathrm{HOMO}}-E_{\mathrm{g}}\right] \mathrm{eV}
$$

\subsection{Material Syntheses}

The manipulations involving air-sensitive reagents were performed in an atmosphere of dry Argon (Ar). The chemicals and solvents, unless otherwise specified, were purchased from Aladdin, Aldrich or Acros, and used without further purification.

\subsubsection{Synthesis of (4-(9H-Carbazol-9-yl)phenyl)diphenylphosphine Sulfide (CzPhPS)}

9-(4-Bromophenyl)-9H-carbazole ( $3.2 \mathrm{~g}, 10.0 \mathrm{mmol}, 1.0$ equivalent (eq.)) was dissolved in $50 \mathrm{~mL}$ anhydrous THF under $\mathrm{Ar}$ and cooled to $-78^{\circ} \mathrm{C}$ in a dry ice/acetone bath. $n$-BuLi (7.5 mL, $12.0 \mathrm{mmol}, 1.2$ eq., $1.6 \mathrm{M}$ in hexane) was then added dropwise to give a yellow solution. Two hours later, $2.0 \mathrm{~mL}$ diphenylphosphine chloride $(10.0 \mathrm{mmol}, 1.0 \mathrm{eq}$.) was injected into the system at $-78^{\circ} \mathrm{C}$ and the mixture was stirred at this temperature for $1 \mathrm{~h}$, following which the temperature was allowed to increase to ambient. After stirring overnight, the reaction was quenched with $50 \mathrm{~mL}$ water and extracted by dichloromethane $(3 \times 50 \mathrm{~mL})$. The organic layer was dried with anhydride $\mathrm{Na}_{2} \mathrm{SO}_{4}$, and the solvent was evaporated under reduced pressure. The resulting solid resides were dissolved in dichloromethane $(50 \mathrm{~mL})$ for sulfurization by treating with an excessive amount of sulfur $(1.0 \mathrm{~g}, 30 \mathrm{mmol}$, 3.0 eq.). After stirring overnight at room temperature, the solvent was removed, and the crude product was purified by a column chromatography to obtain a pure white solid. Yield: $2.9 \mathrm{~g}, 63.0 \% .{ }^{1} \mathrm{H}$ NMR $\left(400 \mathrm{MHz}, \mathrm{CDCl}_{3}, \mathrm{ppm}\right) \delta 8.15(\mathrm{~d}, J=8.0 \mathrm{~Hz}, 2 \mathrm{H})$, $7.98(\mathrm{dd}, J=12.0,12.0 \mathrm{~Hz}, 2 \mathrm{H}), 7.86(\mathrm{~m}, 4 \mathrm{H}), 7.71(\mathrm{dd}, J=8.0,4.0 \mathrm{~Hz}, 2 \mathrm{H}), 7.57(\mathrm{~m}, 8 \mathrm{H}), 7.44$ $(\mathrm{m}, 2 \mathrm{H}), 7.33(\mathrm{~m}, 2 \mathrm{H}) .{ }^{13} \mathrm{C}$ NMR $\left(100 \mathrm{MHz}, \mathrm{CDCl}_{3}, \mathrm{ppm}\right) \delta 140.94,140.19,134.03,133.92$, 133.05, 132.39, 132.28, 132.20, 132.13, 131.83, 131.81, 131.28, 128.78, 128.66, 126.52, 126.39, $126.18,123.81,120.61,120.47,109.77$. HRMS (EI): $m / z$ calcd for $\mathrm{C}_{30} \mathrm{H}_{22} \mathrm{NPS}[\mathrm{M}+\mathrm{H}]^{+}$: 460.1289; found: 460.1284 . 


\subsubsection{Synthesis of Bis(4-(9H-carbazol-9-yl)phenyl)(phenyl)phosphine Sulfide (DCzPhPS)}

DCzPhPS was prepared under the identical synthetic conditions described in the preparation of CzPhPS using 9-(4-bromophenyl)-9H-carbazole ( $3.2 \mathrm{~g}, 10.0 \mathrm{mmol}, 2.0 \mathrm{eq}$.), $n$-BuLi (7.5 mL, $12 \mathrm{mmol}$, 2.4 eq., $1.6 \mathrm{M}$ in hexane), phenylphosphane dichloride $(0.7 \mathrm{~mL}$, $5.0 \mathrm{mmol}, 1.0$ eq.) and sulfur ( $0.5 \mathrm{~g}, 15 \mathrm{mmol}, 3.0 \mathrm{eq}$.). Yield: $1.8 \mathrm{~g}$ of white power $(57.6 \%)$. ${ }^{1} \mathrm{H}$ NMR $\left(400 \mathrm{MHz}, \mathrm{CDCl}_{3}, \mathrm{ppm}\right) \delta 8.16(\mathrm{~d}, J=8.0 \mathrm{~Hz}, 4 \mathrm{H}), 8.07(\mathrm{~m}, 4 \mathrm{H}), 7.97(\mathrm{~m}, 2 \mathrm{H})$, $7.79(\mathrm{dd}, J=4.0 \mathrm{~Hz}, 4 \mathrm{H}), 7.62(\mathrm{~m}, 3 \mathrm{H}), 7.55(\mathrm{~d}, J=8.0 \mathrm{~Hz}, 4 \mathrm{H}), 7.46(\mathrm{~m}, 4 \mathrm{H}), 7.35(\mathrm{~m}, 4 \mathrm{H})$. ${ }^{13} \mathrm{C}$ NMR $\left(100 \mathrm{MHz}, \mathrm{CDCl}_{3}\right) \delta 141.17,140.17,134.08,133.97,132.43,132.33,132.05,131.75$, $130.89,128.96,128.84,126.65,126.52,126.22,123.87,120.69,120.51,109.78$. HRMS (EI): $m / z$ calcd for $\mathrm{C}_{42} \mathrm{H}_{29} \mathrm{~N}_{2} \mathrm{PS}[\mathrm{M}+\mathrm{H}]^{+}$: 625.1867; found: 625.1865 .

\subsubsection{Synthesis of Tris(4-(9H-carbazol-9-yl)phenyl)phosphine Sulfide (TCzPhPS)}

TCzPhPS was prepared under the identical synthetic conditions described in the preparation of CzPhPS using 9-(4-bromophenyl)-9H-carbazole (2.4 g, $7.5 \mathrm{mmol}, 3.0 \mathrm{eq}$.), $n$-BuLi (5.0 mL, $8.0 \mathrm{mmol}, 3.2 \mathrm{eq} .1 .6 \mathrm{M}$ in hexane), triethyl phosphite ( $0.43 \mathrm{~mL}, 2.5 \mathrm{mmol}$, 1.0 eq.) and sulfur $\left(0.25 \mathrm{~g}, 7.5 \mathrm{mmol}, 3.0\right.$ eq.). Yield: $1.2 \mathrm{~g}$ of white power $(60.8 \%) .{ }^{1} \mathrm{H}$ $\operatorname{NMR}\left(400 \mathrm{MHz}, \mathrm{CDCl}_{3}, \mathrm{ppm}\right) \delta 8.20(\mathrm{~m}, 12 \mathrm{H}), 7.86(\mathrm{~m}, 6 \mathrm{H}), 7.59(\mathrm{~d}, J=8.0 \mathrm{~Hz}, 6 \mathrm{H})$, $7.47(\mathrm{~m}, 6 \mathrm{H}), 7.36(\mathrm{t}, J=8.0 \mathrm{~Hz}, 6 \mathrm{H}) ;{ }^{13} \mathrm{C}$ NMR $\left(100 \mathrm{MHz}, \mathrm{CDCl}_{3}, \mathrm{ppm}\right) \delta 141.46,140.15$, 134.13, 131.39, 130.52, 126.78, 126.27, 123.93, 120.78, 109.78; HRMS (EI): $m / z$ calcd for $\mathrm{C}_{54} \mathrm{H}_{36} \mathrm{~N}_{3} \mathrm{PS}[\mathrm{M}+\mathrm{H}]^{+}$: 790.2446; found: 790.2448.

\subsection{Device Fabrication and Characterization}

Typically, ITO-coated glass substrates were etched, patterned, and washed by ultrasonic cleaner with detergent, deionized water, acetone, and ethanol in turn. Organic layers were deposited by high-vacuum (10 $10^{-6}$ Torr) thermal evaporation at a rate of $0.1-0.2 \mathrm{~nm} \mathrm{~s}^{-1}$. The layer thickness and the deposition rate were monitored in situ by an oscillating quartz thickness monitor. The devices were measured after fabrication without encapsulation under an ambient atmosphere at room temperature. EL spectra of the devices were measured by a PR655 spectra scan spectrometer. The luminance-voltage and current-voltage characteristics were recorded using an optical power meter and a Keithley 2602 voltage current source.

\section{Conclusions}

In conclusion, we successfully synthesized three novel bipolar hosts, CzPhPS, DCzPhPS, and TCzPhPS, by using triphenylphosphine sulfide as an electron-deficient unit and adopting mono-, di-, and tri-substituted carbazoles with tunable hole-transport ability as electron-donating groups. The developed hosts show the outstanding thermal stability with the $T_{\mathrm{d}}$ and $T_{\mathrm{m}}$ surpassing $350{ }^{\circ} \mathrm{C}$ and $200{ }^{\circ} \mathrm{C}$, respectively. In addition, the absorption and emission spectra of the three compounds are highly similar, indicating the quantity of carbazoles does not change their intermolecular charge transfer feature. The high $E_{\mathrm{T}}$ levels of $\sim 3.0 \mathrm{eV}$ render these compounds suitable as the hosts for blue OLEDs. From CzPhPS to TCzPhPS, as the number of constructed carbazole units increases, the performance of their PhOLEDs becomes worse and worse with the maximum EQE decreasing from $17.5 \%$ to $7.2 \%$, which can be ascribed to the deteriorated device's charge transport balance. The current work is enlightening for designing bipolar hosts to achieve high-performance blue PhOLEDs.

Supplementary Materials: The following are available online, Figures S1-S6: NMR spectra, Figures S7-S9: mass spectra, Figure S10: phosphorescence spectra.

Author Contributions: Conceptualization, G.X., H.L. and Y.T.; material synthesis and characterization, G.X., Y.C., X.X., X.Z. and C.L.; device fabrication, J.W.; writing-original draft preparation, G.X.; writing-review and editing, Y.T., H.L. and R.C. All authors have read and agreed to the published version of the manuscript. 
Funding: This research was funded in part by the National Natural Science Foundation of China, 22075149 and 62075102, the Jiangsu Specially-Appointed Professor Plan, the Six Talent Plan of Jiangsu Province, XCL-049, Natural Science Fund for Colleges and Universities in Jiangsu Province, 20KJB430001, Scientific Starting Fund from Nanjing University of Posts and Telecommunications (NUPTSF), NY220151, NY219007 and NY217140, China Postdoctoral Science Foundation funded project, 2018M642284, and 1311 Talents Program of Nanjing University of Posts and Telecommunications (Dingshan).

Data Availability Statement: The data presented in this study are available on request from the corresponding author.

Conflicts of Interest: The authors declare no conflict of interest.

Sample Availability: Samples of CzPhPS, DCzPhPS, and TCzPhPS are available from the authors.

\section{References}

1. Ma, Y.; Zhang, H.; Shen, J.; Che, C. Electroluminescence from triplet metal-ligand charge-transfer excited state of transition metal complexes. Synth. Met. 1998, 94, 245-248. [CrossRef]

2. Tang, X.; Cui, L.S.; Li, H.C.; Gillett, A.J.; Auras, F.; Qu, Y.K.; Zhong, C.; Jones, S.T.E.; Jiang, Z.Q.; Friend, R.H.; et al. Highly efficient luminescence from space-confined charge-transfer emitters. Nat. Mater. 2020, 19, 1332-1338. [CrossRef]

3. Li, T.-Y.; Wu, J.; Wu, Z.-G.; Zheng, Y.-X.; Zuo, J.-L.; Pan, Y. Rational design of phosphorescent iridium(III) complexes for emission color tunability and their applications in OLEDs. Coord. Chem. Rev. 2018, 374, 55-92. [CrossRef]

4. Yook, K.S.; Lee, J.Y. Small molecule host materials for solution processed phosphorescent organic light-emitting diodes. Adv. Mater. 2014, 26, 4218-4233. [CrossRef] [PubMed]

5. Xie, G.; Li, X.; Chen, D.; Wang, Z.; Cai, X.; Chen, D.; Li, Y.; Liu, K.; Cao, Y.; Su, S.-J. Evaporation- and solution-process-feasible highly efficient thianthrene-9, $9^{\prime}, 10,10^{\prime}$-tetraoxide-based thermally activated delayed fluorescence emitters with reduced efficiency roll-off. Adv. Mater. 2016, 28, 181-187. [CrossRef] [PubMed]

6. Jiang, H.; Tao, Y.; Jin, J.; Dai, Y.; Xian, L.; Wang, J.; Wang, S.; Chen, R.; Zheng, C.; Huang, W. Resonance-driven dynamically bipolar organic semiconductors for high-performance optoelectronic applications. Mater. Horiz. 2020, 7, 3298-3304. [CrossRef]

7. Su, S.-J.; Sasabe, H.; Takeda, T.; Kido, J. Pyridine-containing bipolar host materials for highly efficient blue phosphorescent OLEDs. Chem. Mater. 2008, 20, 1691-1693. [CrossRef]

8. Li, Y.; Liu, J.-Y.; Zhao, Y.-D.; Cao, Y.-C. Recent advancements of high efficient donor-acceptor type blue small molecule applied for OLEDs. Mater. Today 2017, 20, 258-266. [CrossRef]

9. Zhang, Y.; Liu, C.; Hang, X.-C.; Xue, Q.; Xie, G.; Zhang, C.; Qin, T.; Sun, Z.; Chen, Z.-K.; Huang, W. Phenylquinoline fused cyclic derivatives as electron acceptors of exciplex forming hosts for solution-processable red phosphorescent OLEDs. J. Mater. Chem. C 2018, 6, 8035-8041. [CrossRef]

10. Jang, H.J.; Braveenth, R.; Raagulan, K.; Choi, S.Y.; Park, Y.H.; Oh, S.B.; Bae, I.-J.; Kim, B.M.; Wu, Q.; Kim, M.; et al. Dibenzothiophene dioxide-benzofuro carbazole based bipolar host material for yellow and red phosphorescent OLEDs. Dyes Pigm. 2020, 182, 108697. [CrossRef]

11. Tian, Q.S.; Yuan, S.; Shen, W.S.; Zhang, Y.L.; Wang, X.Q.; Kong, F.C.; Liao, L.S. Multichannel effect of triplet excitons for highly efficient green and red phosphorescent OLEDs. Adv. Opt. Mater. 2020, 8, 2000556. [CrossRef]

12. Su, S.-J.; Cai, C.; Kido, J. Three-carbazole-armed host materials with various cores for RGB phosphorescent organic light-emitting diodes. J. Mater. Chem. 2012, 22, 3447-3456. [CrossRef]

13. Wang, Y.; Yun, J.H.; Wang, L.; Lee, J.Y. High triplet energy hosts for blue organic light-emitting diodes. Adv. Funct. Mater. 2020, 31, 2008332. [CrossRef]

14. Li, H.; Li, H.; Zhi, Y.; Wang, J.; Tang, L.; Tao, Y.; Xie, G.; Zheng, C.; Huang, W.; Chen, R. Multiple $\sigma-\pi$ conjugated molecules with selectively enhanced electrical performance for efficient solution-processed blue electrophosphorescence. Adv. Opt. Mater. 2019, 7, 1901124. [CrossRef]

15. Jin, J.; Tao, Y.; Jiang, H.; Chen, R.; Xie, G.; Xue, Q.; Tao, C.; Jin, L.; Zheng, C.; Huang, W. Star-shaped boron-containing asymmetric host materials for solution-processable phosphorescent organic light-emitting diodes. Adv. Sci. 2018, 5, 1800292. [CrossRef] [PubMed]

16. Li, Y.; Wang, Z.; Li, X.; Xie, G.; Chen, D.; Wang, Y.-F.; Lo, C.-C.; Lien, A.; Peng, J.; Cao, Y.; et al. Highly efficient spiro[fluorene-9,9'thioxanthene] core derived blue emitters and fluorescent/phosphorescent hybrid white organic light-emitting diodes. Chem. Mater. 2015, 27, 1100-1109. [CrossRef]

17. Xie, G.; Chen, D.; Li, X.; Cai, X.; Li, Y.; Chen, D.; Liu, K.; Zhang, Q.; Cao, Y.; Su, S.-J. Polarity-tunable host materials and their applications in thermally activated delayed fluorescence organic light-emitting diodes. ACS Appl. Mater. Interfaces 2016, 8, 27920-27930. [CrossRef]

18. Zhao, Z.; Yu, G.; Chang, Q.; Liu, X.; Liu, Y.; Wang, L.; Liu, Z.; Bian, Z.; Liu, W.; Huang, C. Carbazolylphosphines and carbazolylphosphine oxides: Facilely synthesized host materials with tunable mobilities and high triplet energy levels for blue phosphorescent OLEDs. J. Mater. Chem. C 2017, 5, 7344-7351. [CrossRef] 
19. Han, C.; Zhu, L.; Li, J.; Zhao, F.; Zhang, Z.; Xu, H.; Deng, Z.; Ma, D.; Yan, P. Highly efficient multifluorenyl host materials with unsymmetrical molecular configurations and localized triplet states for green and red phosphorescent devices. Adv. Mater. 2014, 26, 7070-7077. [CrossRef]

20. Tao, Y.; Xiao, J.; Zheng, C.; Zhang, Z.; Yan, M.; Chen, R.; Zhou, X.; Li, H.; An, Z.; Wang, Z.; et al. Dynamically adaptive characteristics of resonance variation for selectively enhancing electrical performance of organic semiconductors. Angew. Chem. Int. Ed. 2013, 52, 10491-10495. [CrossRef] [PubMed]

21. Han, C.; Zhang, Z.; Xu, H.; Li, J.; Xie, G.; Chen, R.; Zhao, Y.; Huang, W. Controllably tuning excited-state energy in ternary hosts for ultralow-voltage-driven blue electrophosphorescence. Angew. Chem. Int. Ed. 2012, 51, 10104-10108. [CrossRef]

22. Han, C.; Zhang, J.; Ma, P.; Yang, W.; Xu, H. Host engineering based on multiple phosphorylation for efficient blue and white TADF organic light-emitting diodes. Chem. Eng. J. 2021, 405, 126986. [CrossRef]

23. Song, X.; Xu, H. Pure-organic phosphine oxide luminescent materials. J. Inf. Disp. 2020, 21, 149-172. [CrossRef]

24. Wada, A.; Yasuda, T.; Zhang, Q.; Yang, Y.S.; Takasu, I.; Enomoto, S.; Adachi, C. A host material consisting of a phosphinic amide directly linked donor-acceptor structure for efficient blue phosphorescent organic light-emitting diodes. J. Mater. Chem. C 2013, 1, 2404-2407. [CrossRef]

25. Tao, Y.; Xu, L.; Zhang, Z.; Chen, R.; Li, H.; Xu, H.; Zheng, C.; Huang, W. Achieving optimal self-adaptivity for dynamic tuning of organic semiconductors through resonance engineering. J. Am. Chem. Soc. 2016, 138, 9655-9662. [CrossRef] [PubMed]

26. Li, H.; Tao, Y.; Zhi, Y.; Chen, R.; Li, H.; Xing, G.; Xu, S.; Huang, W. Evoking non-bonding S- $\pi$ interaction by aryl phosphine sulfide for selectively enhanced electronic property of organic semiconductors. Chem. Eng. J. 2020, 380, 122562. [CrossRef]

27. Tao, Y.; Chen, R.; Li, H.; Yuan, J.; Wan, Y.; Jiang, H.; Chen, C.; Si, Y.; Zheng, C.; Yang, B.; et al. Resonance-activated spin-flipping for efficient organic ultralong room-temperature phosphorescence. Adv. Mater. 2018, 30, 1803856. [CrossRef]

28. Yook, K.S.; Lee, J.Y. Organic materials for deep blue phosphorescent organic light-emitting diodes. Adv. Mater. 2012, 24, 3169-3190. [CrossRef]

29. Cardona, C.M.; Li, W.; Kaifer, A.E.; Stockdale, D.; Bazan, G.C. Electrochemical considerations for determining absolute frontier orbital energy levels of conjugated polymers for solar cell applications. Adv. Mater. 2011, 23, 2367-2371. [CrossRef] [PubMed]

30. Li, H.; Jin, J.; Xiang, Y.; Zhang, Y.; Tao, Y.; Li, M.; Xue, Q.; Xie, G.; Huang, W.; Chen, R. Resonance hosts for high efficiency solution-processed blue and white electrophosphorescent devices. Sci. China Chem. 2020, 63, 1645-1651. [CrossRef]

31. Li, X.L.; Xie, G.; Liu, M.; Chen, D.; Cai, X.; Peng, J.; Cao, Y.; Su, S.-J. High-efficiency WOLEDs with high color-rendering index based on a chromaticity-adjustable yellow thermally activated delayed fluorescence emitter. Adv. Mater. 2016, 28, 4614-4619. [CrossRef]

32. Chaskar, A.; Chen, H.-F.; Wong, K.-T. Bipolar host materials: A chemical approach for highly efficient electrophosphorescent devices. Adv. Mater. 2011, 23, 3876-3895. [CrossRef]

33. Wang, X.; Wang, S.; Ma, Z.; Ding, J.; Wang, L.; Jing, X.; Wang, F. Solution-processible 2,2'-dimethyl-biphenyl cored carbazole dendrimers as universal hosts for efficient blue, green, and red phosphorescent OLEDs. Adv. Funct. Mater. 2014, 24, 3413-3421. [CrossRef]

34. Liu, D.; Wang, F.; Yao, R. Molecular evolution of host materials by regular tuning N/P ratio for high-performance phosphorescence organic light-emitting. J. Mater. Chem. C 2018, 6, 7839-7846. [CrossRef]

35. Oner, S.; Aydemir, M.; Yesil, F.; Sahin, C.; Varlikli, C. Synthesis, photophysical and electrochemical properties of novel carbazoletriazine based high triplet energy, solution-processable materials. Dyes Pigm. 2018, 159, 92-99. [CrossRef] 